\title{
RESPOSTAS JURÍDICAS E POLÍTICAS CONTRA A CORRUPC̣ÃO SISTÊMICA
}

https://doi.org/10.1590/2317-6172202119

\section{INTRODUÇÃO}

Em agosto de 2020, um simpósio, organizado conjuntamente pela Faculdade de Direito da Universidade de Toronto e pela Escola de Direito da Fundação Getulio Vargas, reuniu um grupo interdisciplinar de acadêmicos para refletir sobre as promessas e os limites das medidas jurídicas e políticas contra a corrupção sistêmica. Os resultados desse simpósio estão agora compilados em duas publicações: este número especial da Revista Direito GV e uma edição especial do University of Toronto Law Journal (DAVIS et al., 2021).

O simpósio foi inspirado em grande parte pela Operação Lava Jato, talvez o maior caso anticorrupção da história. A Operação Lava Jato desencadeou debates extensos no Brasil e em outros países da América Latina acerca de sua legalidade e suas ramificações políticas e econômicas (LAGUNES e SVEJNAR, 2020; PRADO e MACHADO, 2021; MARONA e KERCHE, 2020; TAYLOR, 2019; SÁ E SILVA, 2020; PIMENTA, 2020; OCANTOS e HIDALGO, 2019). A Operação Lava Jato também levanta questões mais amplas sobre o papel do direito - especialmente do direito penal - no combate à corrupção sistêmica.

Esta introdução fornece um panorama dos artigos publicados neste número especial e no University of Toronto Law Journal. Começamos com um panorama do problema e das possíveis respostas jurídicas a ele. Depois, passamos às contribuições que discutem as duas características mais salientes de muitas medidas contra a corrupção sistêmica: a ênfase no direito penal e o uso de múltiplas instituições de controle. Em seguida, consideramos alternativas às respostas jurídicas, especificamente intervenções eleitorais e de outras esferas estatais. Finalmente, discutimos as considerações que esta coletânea de artigos oferece quanto aos esforços de avaliar e aprender com as medidas contra a corrupção sistêmica.

\section{PANORAMA do PROBlema e POTENCIAIS RESPOSTAS}

Davis (2021a) abre este número especial com uma avaliação da literatura sobre o papel que o direito desempenha na luta contra a corrupção sistêmica. O autor começa identificando as características definidoras da corrupção sistêmica e sugere que a corrupção pode variar em termos de prevalência, causas ou percepções, e que pode ser mais ou menos sistêmica em cada uma dessas áreas. Portanto, a corrupção pode ser sistêmica porque é disseminada ou persistente. Alternativamente, pode ser sistêmica porque é causada por características identificáveis das instituições de uma sociedade, em contraste com as decisões idiossincráticas descoordenadas de atores individuais. Aqui, Davis traça mais uma distinção entre a corrupção que envolve o comprometimento de sistemas concebidos para 
controlar a corrupção (subversiva) e a corrupção causada por características de outras instituições (estrutural). Finalmente, o rótulo "corrupção sistêmica" às vezes indica que um grupo específico de pessoas percebe que a corrupção é disseminada, persistente, subversiva ou estrutural; em outras palavras, que se tornou normalizada.

O restante do ensaio de Davis critica contribuições recentes à literatura que questionam a eficácia de respostas jurídicas contra a corrupção sistêmica, i.e., a aplicação de proibições legais explícitas por instituições jurídicas convencionais. Ele sugere que, independentemente de como a corrupção sistêmica seja definida, respostas jurídicas provavelmente desempenham um papel importante no seu controle. Como? Dada a variedade de fenômenos abarcados pelo conceito de corrupção sistêmica, Davis afirma que o papel que tais respostas podem desempenhar varia segundo a natureza do problema. Mais investigações e sanções são medidas razoáveis contra a corrupção disseminada ou persistente. Recrutar agências adicionais de controle é uma resposta apropriada contra a corrupção subversiva. Finalmente, estratégias jurídicas concebidas deliberadamente para influenciar a opinião popular podem ser uma medida apropriada contra a corrupção normalizada. $\mathrm{O}$ autor segue analisando as condições que têm probabilidade de determinar o apelo de cada uma dessas estratégias, reconhecendo que os méritos de cada estratégia dependem do contexto. Também reconhece que reformas que visam ao incremento de punições não podem ser a única medida contra a corrupção sistêmica.

Esse quadro analítico prepara o terreno para algumas contribuições ao simpósio. Por exemplo, muitas das contribuições examinam um ou mais dos três principais tipos de respostas jurídicas no contexto da Operação Lava Jato: Prado e Machado (2021) documentam o uso heterodoxo de instrumentos jurídicos; Prado e Pimenta (2021), Jones e Pereira Neto (2021), Ferreira (2021) e Pimenta e Venturini (2021) mapeiam a mobilização de múltiplas instituições de aplicação do direito ("multiplicidade institucional”); e Limongi (2021) mostra como as limitações das abordagens convencionais da aplicação do direito penal levaram juízes e promotores a um esforço deliberado de influenciar a opinião popular a seu favor e em apoio a um projeto concebido para ampliar a margem de discricionariedade das agências de aplicação do direito.

\section{0 PAPEL DO DIREITO PENAL}

Algumas contribuições ao simpósio lidam com a resposta mais comum contra a corrupção sistêmica: o direito penal. Esse tipo de resposta costuma envolver reformas voltadas a expandir proibições, remover obstáculos processuais à investigação e ao processo, reforçar sanções ou aumentar os recursos dedicados à aplicação do direito. O direito penal é a ferramenta mais poderosa disponível para o Estado, com sanções que envolvem privação de liberdade, medidas cautelares severas (como prisão preventiva e confisco de bens) e amplos poderes investigativos. 
Esse repertório dá à polícia e aos promotores uma vantagem considerável sobre os réus, tanto no julgamento quanto na obtenção de soluções negociadas, e presumivelmente cria poderosos incentivos dissuasórios. Ademais, Chiao (2021) propõe que, em cenários de corrupção sistêmica, o direito penal pode desempenhar um papel importante na estabilização da norma de comportamento honesto.

Contudo, há várias razões para duvidar do recurso ao direito penal como medida contra a corrupção sistêmica. Inicialmente, há a preocupação quanto à sua eficácia: a corrupção sistêmica pode cooptar, subjugar, subverter ou paralisar o sistema jurídico. Agentes responsáveis pela aplicação do direito podem estar comprometidos. Além disso, os sistemas de justiça penal em geral são concebidos para lidar com casos individuais, nos quais há um autor, uma vítima e um ato que pode ser ligado diretamente a um resultado específico. Problemas sistêmicos e coletivos não se enquadram nesse padrão, e as tentativas de adaptar processos criminais para solucionar problemas sistêmicos obtiveram resultados discutíveis. Ademais, a expansão de proibições jurídicas, maiores poderes investigativos e sanções mais severas podem ser usados por instituições de controle - com poucas chances de responsabilização -, para reprimir adversários políticos ou grupos marginalizados, o que é contrário aos princípios da democracia, da legitimidade e da equidade processual. Uma atenção exacerbada ao sistema de justiça penal pode fomentar o populismo penal, com dano potencial ao devido processo legal e ao Estado de direito (ROBERTS et al., 2002). Medidas penais severas também podem sair pela culatra: líderes corruptos podem explorar processos abertos contra eles usando alegações de perseguição ou inequidade para insuflar seus apoiadores e reverter a responsabilização.

A Operação Lava Jato é exemplo paradigmático de uma tentativa de adaptar o direito penal para lutar contra a corrupção sistêmica. Como mostram Prado e Machado (2021), os procedimentos penais usados pela primeira vez durante a Operação Lava Jato foram cruciais para revelar informações. Eles também resultaram em medidas jurídicas sem precedentes, incluindo a condenação penal de políticos e empresários do alto escalão, assim como a recuperação de valores substanciais desviados. É interessante que a operação foi facilitada por reformas institucionais introduzidas poucos anos antes por um governo liderado pelo Partido dos Trabalhadores, do qual muitos membros acabaram sendo alvo da Operação Lava Jato. Essas reformas reforçaram e incrementaram a independência da Polícia Federal e do Ministério Público Federal e incentivaram o uso de forças-tarefa que envolveram múltiplas agências.

Outra característica da Operação Lava Jato foi a maneira inédita como os promotores e juízes cortejaram a mídia e buscaram a atenção do público. A fim de promover transparência e obter apoio, eles disponibilizaram decisões e documentos do caso em um site aberto, o que facilitou o acesso do público. Também fizeram uso proativo de veículos de mídia. Prisões, buscas e apreensões, conduções coercitivas e interrogatórios foram difundidos pela mídia e seguidos por 
conferências de imprensa dadas por membros da Polícia Federal e da força-tarefa da Operação Lava Jato, com resultados ambivalentes (PRADO e MACHADO, no prelo).

Além disso, como afirmam Prado e Machado (2021), a Operação Lava Jato introduziu diversas práticas heterodoxas de aplicação do direito, que incluíam ações coordenadas de agentes da Polícia Federal lançadas ao raiar do dia, concebidas para pegar os alvos da investigação de surpresa, além do uso intenso de conduções coercitivas, bem como, em alguns casos, o abuso de instrumentos como acordos de delação e prisões preventivas. Essas práticas levantaram sérias preocupações quanto aos limites do Estado de direito e do devido processo legal. Por conseguinte, a Operação Lava Jato também ilustra as razões da ambivalência acerca das ferramentas do direito penal para lidar com a corrupção sistêmica.

Limongi (2021) ilumina as razões pelas quais os líderes da Operação Lava Jato adotaram as práticas heterodoxas descritas por Prado e Machado (2021) e fizeram um uso tão controverso da mídia. Ele documenta como Sérgio Moro, juiz que presidiu a maioria dos processos da Operação Lava Jato em Curitiba, e o coordenador da Força-Tarefa, o promotor da República Deltan Dallagnol, estavam convencidos de que a corrupção no Brasil era sistêmica, no sentido de que era disseminada entre membros da classe política. Na visão deles, a única forma de combater esse tipo de corrupção era que juízes e promotores se empenhassem em um ataque sistemático - na verdade, uma "cruzada" - contra os políticos corruptos e tentassem ganhar apoio público para sua causa. O autor afirma que a combinação de ações espetaculares, transparência e cooperação com a mídia foi uma estratégia deliberada para atrair atenção e ganhar apoio do público em geral.

Limongi (2021) também questiona a capacidade dos juízes e promotores de administrar os efeitos políticos de grandes julgamentos anticorrupção. Ele afirma que a Operação Lava Jato fracassou em cumprir as esperanças de Moro e dos promotores de promover mudanças significativas na estrutura política brasileira e na prevalência da corrupção política no alto escalão. Sua narrativa ilumina os limites do direito penal como ferramenta para solucionar um problema sistêmico, como ilustrado pelas práticas heterodoxas adotadas no caso e pela tentativa de promover reformas jurídicas (denominada "10 medidas contra a corrupção"), que teriam como resultado o aumento dos poderes discricionários de juízes e promotores e a limitação de importantes garantias do devido processo legal. Como falharam em seus esforços explícitos de ganhar apoio popular para seu projeto de reformas penais, os procuradores formaram, com apoio de juízes proeminentes, uma coalizão improvisada com o movimento antigovernista pelo impeachment. Por conseguinte, juízes e procuradores da Operação Lava Jato tornaram-se atores políticos, sem ser necessariamente figuras centrais. No fim, Limongi questiona se agentes de aplicação do direito penal e, em particular, juízes e promotores podem e devem desempenhar um papel central em desbaratar a corrupção sistêmica no sistema político. 
Embora a Operação Lava Jato seja um caso de advertência quanto aos riscos do processo penal, isso não significa que uma avaliação crítica das medidas jurídicas contra a corrupção deva dispensar uma estratégia penal. As limitações da responsabilidade penal individual e das sanções punitivas sugerem que um caminho promissor para futuras pesquisas pode ser explorar diferentes combinações entre as respostas jurídicas que às vezes se sobrepõem: preventivas e reativas/punitivas; reformas estruturais combinadas com atribuição de responsabilidade; e sanções que vão de privação de direitos, reparações e multas a medidas comunicativas ou reputacionais. Analisar os méritos dessas combinações alternativas é uma agenda de pesquisa promissora para estudiosos do fenômeno da corrupção. Neste número especial, um método para implementar essas estratégias é discutido mais detidamente: o uso de múltiplas agências de aplicação do direito com autoridades sobrepostas. É o que analisaremos a seguir.

\section{MULTIPLICIDADE INSTITUCIONAL}

Estudar medidas jurídicas contra a corrupção sistêmica envolve necessariamente prestar maior atenção à aplicação do direito (DAVIS, JORGE e MACHADO, 2015), e uma das características mais notáveis das ações de aplicação voltadas à corrupção sistêmica - incluindo a Operação Lava Jato - é a multiplicidade institucional. No contexto do direito anticorrupção, a multiplicidade institucional refere-se a uma situação em que múltiplas instituições do mesmo sistema jurídico têm competência para enfrentar um caso de corrupção (DAVIS, JORGE e MACHADO, 2015; PRADO e CORNELIUS, 2020). Por exemplo, em muitos sistemas jurídicos, o mesmo ato de corrupção pode estar sujeito a sanções administrativas, civis ou penais, e diversas instituições, tanto locais quanto estrangeiras, podem aplicar essas sanções. Uma literatura nascente explora as maneiras positivas e negativas com as quais instituições podem interagir em situações que envolvem multiplicidade institucional (DAVIS, JORGE e MACHADO, 2015; PRADO e CORNELIUS, 2020; PRADO e CARSON, 2016; PRADO, CARSON e CORREA, 2015; MACHADO e PASCHOAL, 2016; MACHADO, 2019).

Prado e Pimenta (2021) contribuem para essa linha de investigação tentando identificar condições nas quais a multiplicidade pode aumentar ou diminuir a probabilidade de denúncia de atos ilícitos. As autoras propõem uma visão granular da corrupção sistêmica em que instituições com sistemas funcionais de accountability podem existir em um ambiente no qual outras instituições são disfuncionais, e vice-versa. Pressupondo que "bolsões de honestidade" podem existir em ambientes corruptos, a multiplicidade institucional pode oferecer um caminho alternativo para um indivíduo que está imerso em corrupção sistêmica e tem informações sobre atos ilícitos (e.g., um denunciante ou réu em potencial). A multiplicidade pode permitir que esse indivíduo busque a responsabilização em outro lugar, contornando bloqueios no sistema. Porém, a multiplicidade também pode ser usada para minar a eficácia do sistema de responsabilização, 
especialmente se as instituições de responsabilização forem “bolsões de corrupção”. Em tal cenário, Prado e Pimenta (2021) discutem o risco de "punição de fachada" (facade enforcement) associado a certas formas de multiplicidade, ou seja, uma ação de aplicação do direito que parece proporcionar responsabilização, mas é eivada de corrupção. Elas oferecem exemplos desses cenários usando estudos de caso do Brasil e exploram certas implicações para as políticas públicas: por exemplo, quando e como multiplicidade, centralização ou coordenação pode ser desejável, assim como os benefícios associados a cada uma dessas estratégias. A hipótese que elas apresentam relativiza as alegações de que a multiplicidade sempre ameaça as políticas anticorrupção e abre espaço para uma avaliação mais equilibrada das dimensões positivas e negativas da multiplicidade institucional em diferentes contextos.

Na mesma linha, Jones e Pereira Neto (2021) fornecem uma análise aprofundada da Operação Lava Jato, mostrando como ela envolveu um caso de corrupção sistêmica e examinando maneiras de mitigar os efeitos negativos da multiplicidade institucional. Eles afirmam que diversos fatores interligados explicam a natureza sistêmica da corrupção revelada pelas investigações. Primeiro, compras públicas e empresas estatais estavam no centro do escândalo. Um segundo fator era o envolvimento de políticos que indicavam funcionários nas empresas compradoras em troca de lucro pessoal e doações políticas. Terceiro, havia a percepção de um baixo risco de detecção e punição da corrupção. Em seguida, eles mapeiam múltiplos procedimentos penais, civis e administrativos contra indivíduos e empresas relacionados à Operação Lava Jato. Embora esses diversos procedimentos tenham levado a sanções e penas inéditas, também podem ter criado dupla punição (bis in idem). Os autores propõem reformas para promover um alinhamento mais estreito de políticas de compras públicas, anticorrupção e concorrência e uma coordenação mais próxima entre agentes de compras públicas e agências de aplicação do direito. Os autores também consideram as coalizões políticas necessárias para sustentar as reformas que eles sugerem. Reconhecem que, embora as reformas mais estruturais dependam de vontade política, existem algumas reformas mais acessíveis - e.g., mudanças no sistema de compras públicas - que têm menor probabilidade de enfrentar resistência feroz e poderiam ter implicações positivas a curto e longo prazos.

Ferreira (2021) oferece outra perspectiva sobre os efeitos da multiplicidade institucional. Ao invés de discutir como diferentes agências desempenham funções de responsabilização, ela investiga como tribunais e advogados interpretaram uma lei que tentou criar multiplicidade no Brasil, a Lei de Improbidade Administrativa (Lei n. 8.429/1992). A lei introduziu responsabilidade civil por atos corruptos na tentativa de criar uma alternativa a processos penais, que eram vistos como procedimentalmente complexos e com garantias que reduzem a probabilidade de condenação. Ela afirma, no entanto, que os efeitos da Lei de Improbidade foram mistos: apesar de existir condenações, os casos levam muito tempo para ser decididos e a recuperação de ativos é limitada. Ao explicar as razões para esses resultados frustrantes, Ferreira demonstra 
que a ausência de disposições procedimentais claras na lei permitiu interpretações jurídicas que simplesmente seguiam o processo penal ou reproduziam em grande parte as garantias penais. A autora também analisa a batalha pela jurisdição especial para processar autoridades e políticos. Depois de muitos anos de interpretações judiciais contraditórias, que levaram a numerosos recursos, os tribunais chegaram à conclusão de que salvaguardas processuais são de natureza civil e de que não há jurisdição especial para cargos políticos eleitos ou nomeados. O artigo conclui indicando que ainda há outras questões de equidade processual a serem resolvidas, como o potencial bis in idem gerado pelos casos de multiplicidade institucional, uma questão que é constantemente debatida pelos tribunais.

A multiplicidade institucional leva a outras complicações quando a regulação da corrupção assume uma dimensão transnacional. Instituições jurídicas nacionais podem, como dito antes, ter capacidade limitada de efetivamente enfrentar a corrupção sistêmica. Instituições estrangeiras podem atuar como um complemento, pois podem trazer novos recursos e não estão imersas no mesmo ambiente de economia política (DAVIS, JORGE e MACHADO, 2015). No entanto, há questões importantes sobre a eficácia e a legitimidade de agências estrangeiras. Duas contribuições ao projeto ressaltam algumas dessas questões.

A aplicação transnacional do direito tem sido impulsionada pela aplicação extensiva da Lei de Práticas Corruptas Estrangeiras (Foreign Corrupt Practices Act - FCPA) dos Estados Unidos, uma lei voltada aos casos de suborno de funcionários públicos estrangeiros que tem alcance extraterritorial significativo. Acorn (2021) mostra que há muitas questões abertas sobre o uso da FCPA contra empresas estrangeiras. Por que os Estados Unidos estão dispostos a incorrer nos custos de policiar a conduta de empresas não estadunidenses no exterior? Quais fatores influenciam o exercício de sua jurisdição? O artigo examina cuidadosamente o corpo de pesquisa interdisciplinar existente sobre o assunto, mapeando estudos que consideram a influência de contextos históricos, como oportunidades jurídicas e políticas, e as motivações por trás da aplicação da FCPA. Com base nesse mapa, Acorn ressalta desafios e lacunas metodológicas na literatura existente e aponta potenciais caminhos para a pesquisa futura. Ela conclui sugerindo que novos estudos devem incluir pesquisa qualitativa e continuar a explorar a relação entre aplicação da lei, política externa dos Estados Unidos e política internacional.

A dimensão transnacional da multiplicidade institucional também é analisada por Pimenta e Venturini (2021), que enfocam a cooperação transnacional em casos de suborno por meio de uma análise aprofundada de acordos negociados envolvendo a Odebrecht. Acordos negociados foram uma ferramenta central em iniciativas anticorrupção, em casos nacionais e transnacionais. Analisando acordos da Odebrecht no Brasil, na Suíça, nos Estados Unidos, na República Dominicana e no Peru, o artigo articula uma distinção entre cooperação investigativa, cooperação na imposição de sanções e cooperação na execução dos acordos. Após descrever as características 
de cada ferramenta, os autores exploram os efeitos - positivos e negativos - de empregá-las em um contexto dinâmico que envolve múltiplos países buscando acordos com os mesmos réus, simultânea ou sucessivamente. A cooperação investigativa entre autoridades de diferentes países pode auxiliar a negociação de acordos conjuntos dessas mesmas autoridades. Em contraste, se um acordo conjunto for assinado apenas por poucos países afetados, ele pode ter impacto negativo subsequente na cooperação investigativa com autoridades que não fizeram parte das negociações. Essas distinções podem servir de base para uma possível agenda de pesquisa sobre desenho regulatório em contextos que envolvem cooperação transnacional e multiplicidade institucional. À medida que mais países adotam acordos como parte de suas ferramentas anticorrupção, mais pesquisas são necessárias sobre como os diferentes tipos de acordos interagem, e como diferentes instituições e normas podem inesperadamente enfraquecer ou empoderar diversos atores.

\section{Respostas políticas}

Instituições jurídicas não são os únicos atores capazes de controlar a corrupção. Na verdade, existem certos contextos em que essas espécies de respostas jurídicas provavelmente são inúteis. Por exemplo, os esforços para aumentar a punição serão infrutíferos se instituições jurídicas relevantes tiverem sido subvertidas. Também haverá contextos em que medidas jurídicas trarão resultados provavelmente inferiores às alternativas. Por exemplo, reformas estruturais parecem a medida lógica contra formas estruturais de corrupção. Ademais, na medida em que há preocupações quanto à legitimidade de agentes do Estado não eleitos visarem atores políticos que ocupam cargos eletivos, pode ser preferível que agentes eleitos, ou eleitores, assumam a liderança nos esforços contra a corrupção. Duas contribuições ao nosso simpósio analisam respostas políticas, em contraste com as jurídicas, explorando os papéis potenciais da separação de poderes (DA ROS e TAYLOR, 2021) e das eleições (LAGUNES et al., 2021) para responsabilizar atores políticos do alto escalão.

Da Ros e Taylor (2021) contestam a pressuposição recorrente de que os freios e contrapesos e a separação de poderes sempre são instrumentos eficazes para coibir o abuso de poder e, portanto, a corrupção. Eles o fazem traçando uma distinção entre esses dois conceitos correlatos. Afirmam que a separação de poderes é um conceito mais amplo que pode abarcar diferentes tipos de freios e contrapesos, incluindo formas abusivas ou impróprias, além do uso do próprio Estado de direito como arma política. Para explorar as implicações da distinção conceitual, eles discutem a dinâmica da separação de poderes em crises que levaram à remoção de presidentes e usos mais rotineiros de freios e contrapesos. Nem todas essas dinâmicas levam ao controle da corrupção. Os autores salientam o fato de que a ecologia de um regime (i.e., a distribuição do poder econômico e político, o engajamento cívico e as capacidades estatais) determina “como o jogo é jogado". Finalmente, situam os freios e contrapesos como 
variável mediadora entre a ecologia do regime e o controle da corrupção, uma conclusão que serve de alerta a uma visão excessivamente otimista dos freios e contrapesos como receita para controlar a corrupção.

Lagunes et al. (2021) voltam-se para outro mecanismo político que pode ser empregado para coibir a corrupção: as eleições. Um dos efeitos políticos mais acentuados da Operação Lava Jato foi perturbar o equilíbrio que havia prevalecido no sistema político brasileiro desde a promulgação da Constituição de 1988. Isso abriu a arena política a novos atores, muitos dos quais recorreram a um discurso populista antipolítica que incluía acusações veementes de corrupção política. O sentimento anticorrupção abriu caminho para o presidente Jair Bolsonaro chegar ao poder. Lagunes et al. (2021) mostram como, uma vez no poder, Bolsonaro e sua administração fracassaram em manter sua plataforma anticorrupção. A política brasileira, afirmam eles, é permeada pela coexistência de promessas anticorrupção e a persistência de escândalos de corrupção. Todavia, os autores consideram que cumprir as promessas de campanha segue sendo um indicador de se os agentes eleitos continuam respondendo ao eleitorado. Eles monitoram meticulosamente cada promessa feita por Bolsonaro, entre elas, transparência, reformas jurídicas (incluindo seu apoio às 10 medidas contra a corrupção) e o fomento e a preservação de instituições de aplicação do direito. Eles mostram reversão, desmantelamento e/ou indiferença em todas as dimensões. Suas conclusões demonstram que uma campanha política baseada em uma agenda anticorrupção pode não se traduzir em reformas de políticas públicas, o que levanta questões sobre por que a preferência do eleitorado não permanece como força disciplinadora depois que os políticos chegam ao poder.

\section{Monitoramento, aValiação e APRENDIZAdo}

Além de mapear medidas contra a corrupção sistêmica, o simpósio também explorou como avaliar essas medidas. Embora pareça natural examinar sua eficácia na redução da corrupção, Davis (2021b) afirma que, do ponto de vista prático, é muito difícil determinar a eficácia de respostas jurídicas contra um problema como a corrupção. Enfocando o problema do suborno transnacional, o autor identifica três desafios distintos. Primeiro, há o desafio de obter bons dados sobre as características de intervenções jurídicas ou os resultados relevantes. Segundo, pode ser desafiador determinar se existe algum tipo de conexão causal entre essas intervenções e resultados, considerando a variedade e complexidade dos fatores que influenciam um fenômeno como a corrupção. Terceiro, há o desafio de encontrar ou desenvolver a capacidade institucional necessária para avaliar os indícios de eficácia. Todos esses desafios são exacerbados em situações em que há corrupção sistêmica, especialmente quando envolvem multiplicidade institucional e aplicação transnacional do direito. 
Diversas contribuições a esse simpósio sugerem que medidas contra a corrupção sistêmica devem ser avaliadas segundo outros critérios, além da eficácia. Por exemplo, Prado e Machado (2021) avaliam as práticas heterodoxas introduzidas pela Operação Lava Jato em termos de sua compatibilidade com o devido processo legal e o Estado de direito. Por sua vez, Davis (2021a, 2019) defende a avaliação do direito anticorrupção em termos não somente de eficácia, mas também de eficiência, devido processo legal, equidade e legitimidade.

Vieira (2021) avalia a Operação Lava Jato segundo outro critério: seus efeitos sobre a saúde da democracia constitucional brasileira. O autor faz uma intervenção importante em um debate proeminente: se a Operação Lava Jato causou uma crise constitucional no Brasil. Ele conclui que o Brasil sofreu um mal-estar constitucional, mas não uma crise constitucional, e que esse mal-estar não foi causado exclusivamente pela Operação Lava Jato. Em sua rica descrição do contexto político e constitucional em que a Operação Lava Jato ocorreu, Vieira (2021) destaca vários eventos inter-relacionados que levaram a uma crise política e a um mal-estar constitucional. O sistema político e constitucional foi submetido a pressão não somente pela Operação Lava Jato e por seus efeitos colaterais sobre a classe política, mas também por uma decisão do Supremo Tribunal Federal (STF) que fragmentou ainda mais um sistema multipartidário de representação congressual; pela maior onda de agitação social desde a redemocratização, que culminou em protestos por todo o país em 2013; pelo impeachment presidencial e pelas tensões que ele gerou entre o STF e outros ramos do governo, tudo isso durante uma profunda crise econômica com sérias consequências fiscais. O quadro mostra como a tensão crescente motivada por esses eventos levou a uma escalada na reação de importantes atores políticos, que decidiram entregar-se ao que Vieira considera ser um "jogo duro" (constitutional hard ball), em que os atores não violam as normas constitucionais, mas forçam-nas até seus limites (TUSHNET, 2004). A Operação Lava Jato, com suas práticas heterodoxas e na tentativa de cooptar o apoio popular (como descrito em outras contribuições a esse simpósio), fez parte desse jogo. A análise sugere que os excessos da Operação Lava Jato devem ser lidos no contexto desse conflito institucional mais amplo. A análise também tem implicações para além da Operação Lava Jato: a análise de Vieira aponta a importância de melhor mapear e avaliar o impacto político de medidas jurídicas contra a corrupção.

\section{ConClusão}

Este projeto revelou que existe espaço considerável para refinar nosso pensamento sobre respostas alternativas à corrupção sistêmica. O primeiro passo é definir com alguma precisão o que queremos dizer com "corrupção sistêmica”, já que a expressão é usada muitas vezes de forma imprecisa para descrever uma ampla variedade de problemas. Também é importante reconhecer a vasta gama de respostas potenciais contra a corrupção sistêmica - penais e não penais, 
jurídicas e não jurídicas, nacionais e estrangeiras - e como elas podem ser usadas, em combinação ou como alternativas, e como podem interagir. Nesse espírito, os estudos do simpósio sugerem que os efeitos de diferentes medidas dependem não somente da natureza precisa do problema, mas também de complexas interações entre diversos fatores sociais e institucionais. Vários dos estudos, especialmente aqueles focados na Operação Lava Jato, demonstram que os efeitos de tentar remediar a corrupção sistêmica podem ter um longo alcance, atingindo até as estruturas políticas mais fundamentais de uma sociedade. Todos esses tópicos merecem mais estudo.

Dadas a visível complexidade e a especificidade contextual das relações entre intervenções de política pública e corrupção sistêmica, é difícil usar a experiência para extrair lições confiáveis de política pública, especialmente quando a experiência deriva de casos singulares como a Operação Lava Jato. Como sugerem muitas das contribuições ao simpósio, uma forma de levar adiante essa investigação é procurar categorias conceituais que permitam níveis relativamente altos de abstração e generalização. O conceito de multiplicidade institucional, por exemplo, oferece um quadro analítico muito mais amplo que análises jurídicas tradicionais, permitindo comparações que de outra forma não seriam possíveis.

Em relação à análise jurídica, futuros estudos nessa área não serão frutíferos caso se baseiem unicamente em análises doutrinárias tradicionais, i.e., simplesmente levando em conta as disposições legais. No mínimo, é importante considerar as interações entre diferentes ramos do direito e os processos, organizações e personalidades envolvidas na sua aplicação (DAVIS, JORGE e MACHADO, 2015). Também é importante prestar atenção nas interações entre direito e instituições jurídicas, por um lado, e, por outro lado, em fatores sociológicos e políticos, como crenças populares e a distribuição de poder entre diferentes grupos dentro de uma comunidade. Essas análises só podem se beneficiar da interdisciplinaridade que informou nosso simpósio, e que nos permitiu extrair reflexões e gerar um diálogo frutífero entre o direito e outras ciências sociais.

\section{REFERÊNCIAS}

ACORN, Elizabeth. Law and Politics in FCPA Prosecutions of Foreign Corporations. Revista Direito GV, São Paulo, v. 17, n. 2, maio/ago. 2021, e2124. https:// doi.org/10.1590/2317-6172202124

CHIAO, Vincent. Corruption and the Criminal Law: Assurance and Deterrence. University of Toronto Law Journal, Toronto, 2021, no prelo (Special Feature: Law and Systemic Corruption). 
DA ROS, Luciano; TAYLOR, Matthew M. Checks and Balances: The Concept and Its Implications for Corruption. Revista Direito GV, v. 17, n. 2, maio/ago. 2021, e2120. https://doi.org/10.1590/23176172202120

DAVIS, Kevin E. Anti-Corruption Law and Systemic Corruption: The Role of Direct Responses. Revista Direito GV, v. 17, n. 2, maio/ago. 2021a, e2129. https: / / doi.org/10.1590/2317-6172202129

DAVIS, Kevin E. The Limits of Evidence-Based Anti-Bribery Law. University of Toronto Law Journal, Toronto, 2021b, no prelo (Special Feature: Law and Systemic Corruption).

DAVIS, Kevin E. Between Impunity and Imperialism: The Regulation of Transnational Bribery. Oxford: Oxford University Press, 2019.

DAVIS, Kevin E.; JORGE, Guillermo; MACHADO, Maíra Rocha. Transnational Anti-Corruption Law in Action: Cases from Argentina and Brazil. Law \& Social Inquiry, Malden, v. 40, n. 3, p. 664-699, 2015.

DAVIS, Kevin E. et al. Introduction to the Law and Systemic Corruption Special Feature. University of Toronto Law Journal, Toronto, 2021, no prelo (Special Feature: Law and Systemic Corruption).

FERREIRA, Vivian Pereira. When Institutional Multiplicity Backfires: The Battle over the Jurisdiction to Prosecute Politicians for Administrative Improbity in Brazil. Revista Direito GV, São Paulo, v. 17, n. 2, maio/ago. 2021, e2130. https://doi.org/10.1590/2317-6172202130

JONES, Alison; PEREIRA NETO, Caio Mário da Silva. Combating Corruption and Collusion in Public Procurement: Lessons from Operation Car Wash. University of Toronto Law Journal, Toronto, 2021, no prelo (Special Feature: Law and Systemic Corruption).

LAGUNES, Paul; SVEJNAR, Jan (ed.). Corruption and the Lava Jato Scandal in Latin America. Routledge Corruption and Anti-Corruption Studies. New York: Routledge, 2020.

LAGUNES, Paul et al. President Bolsonaro's Promises and Actions on Corruption Control. Revista Direito GV, v. 17, n. 2, maio/ago. 2021, e2121. https:/ / doi.org/10.1590/2317-6172202121

LIMONGI, Fernando. From Birth to Agony: The Political Life of Car Wash (Lava Jato). University of Toronto Law Journal, Toronto, 2021, no prelo (Special Feature: Law and Systemic Corruption).

MACHADO, Maíra Rocha. Independência como indiferença: a face jurídica da multiplicidade institucional em casos de corrupção. Revista Direito, Estado e Sociedade, Rio de Janeiro, n. 55, jul.-dez. 2019. 
MACHADO, Maíra Rocha; PASCHOAL, Bruno. Monitorar, investigar, responsabilizar, sancionar: a multiplicidade institucional em casos de corrupção. Novos Estudos, São Paulo, n. 104, p. 11-36, 2016.

MARONA, Marjorie; KERCHE, Fábio. From the Banestado Case to Operation Car Wash: Building an Anti-Corruption Institutional Framework in Brazil. Dados, Rio de Janeiro, v. 64, n. 3, p. 1-37, 2020.

OCANTOS, Ezequiel Gonzalez; HIDALGO, Viviana Baraybar. Lava Jato beyond Borders: The Uneven Performance of Anti-Corruption Judicial Efforts in Latin America. Taiwan Journal of Democracy, Taiwan, v. 15, n. 1, p. 63-89, 2019.

PIMENTA, Raquel de Mattos. A construção dos acordos de leniência da Lei Anticorrupção. São Paulo: Blucher, 2020.

PIMENTA, Raquel de Mattos;VENTURINI, Otavio. International Cooperation and Negotiated Settlements for Transnational Bribery: A Study of the Odebrecht Case. Revista Direito GV , v. 17, n. 2, maio/ago. 2021, e2131. https://doi.org/10.1590/2317-6172202131

PRADO, Mariana Mota; CARSON, Lindsey D. Brazilian Anti-Corruption Legislation and Its Enforcement: Potential Lessons for Institutional Design. Journal of Self-Governance and Management Economics, v. 4, n. 1, 2016.

PRADO, Mariana Mota; CARSON, Lindsey D.; CORREA, Isabela. The Brazilian Clean Company Act: Using Institutional Multiplicity for Effective Punishment. Osgoode Hall Law Journal, Toronto, v. 53, n. 1, p. 107-163, 2015.

PRADO, Mariana Mota; CORNELIUS, Eduardo. Institutional Multiplicity and the Fight Against Corruption: A Research Agenda for the Brazilian Accountability Network. Revista Direito GV, São Paulo, v. 16, n. 3, p. 1 32, 2020.

PRADO, Mariana Mota; MACHADO, Marta R. de Assis. Uso do direito penal para combater a corrupção: potencial, riscos e limitações da Operação Lava Jato.Trad. Matheus Barros. Revista Direito GV, São Paulo, v. 17, n. 2, maio/ago. 2021.

PRADO, Mariana Mota; MACHADO, Marta R. de Assis. Turning Corruption Trials into Political Tools, with a Little Help from the Media:The Lava Jato Case. In: BOTERO, Sandra; BRINKS, Daniel M.; GONZALESOCANTOS, Ezequiel (coord.). Broken Promises? Taking Stock of the Judicialization of Politics in Latin America. Cambridge: Cambridge University Press, no prelo.

PRADO, Mariana Mota; PIMENTA, Raquel de Mattos. Systemic Corruption and Institutional Multiplicity: Brazilian Examples of a Complex Relationship. University of Toronto Law Journal, Toronto, 2021, no prelo (Special Feature: Law and Systemic Corruption). 
ROBERTS, Julian V. et al. Penal Populism and Public Opinion: Lessons from Five Countries. Oxford: Oxford University Press, 2002.

SÁ E SILVA, Fábio de. From Car Wash to Bolsonaro: Law and Lawyers in Brazil's Illiberal Turn (20142018). Journal of Law and Society, Cardiff, v. 4, n. 1, p. 90-110, 2020.

TAYLOR, Matthew M. The Troubling Strength of Brazilian Institutions in the Face of Scandal. Taiwan Journal of Democracy, Taiwan, v. 15, n. 1, p. 91-111, 2019.

TUSHNET, Mark V. Constitutional Hardball. The John Marshall Law Review, Chicago, v. 37, n. 2, p. 523 $553,2004$.

VIEIRA, Oscar Vilhena. Clash of Powers: Did Operation Car Wash Trigger a Constitutional Crisis in Brazil? University of Toronto Law Journal, Toronto, 2021, no prelo (Special Feature: Law and Systemic Corruption).

\section{Como citar este editorial:}

DAVIS, Kevin E. et al. Respostas jurídicas e políticas contra a corrupção sistêmica. Revista Direito GV, v. 17, n. 2 , maio/ago. 2021, e2119. https://doi.org/ $10.1590 / 2317-6172202119$
Kevin E. Davis

https://orcid.org/0000-0002-9278-6439 Beller Family Professor of Business LaW, Escola de DiReito da Universidade de Nova York. davisklamercury.law.nyu.edu

Marta R. de Assis Machado https://orcid.org/0000-0002-8924-089X Professora na Escola de Direito de São Paulo da Fundação Getulio Vargas (FGV DIREITO SP).

Marta.Machadolafgv.br

Raquel de Mattos Pimenta https://orcid.org/0000-0002-7526-7338 Professora na Escola de Direito de São Paulo da Fundação Getulio VARgas (FGV DIREITO SP). raquel.pimentalafgv.br 
Mariana Mota Prado

https://orcid.org/0000-0002-5555-8859 Professora na Universidade de Toronto, Faculdade de DIREITO.

mariana.pradolutoronto.ca 